\title{
TESTE DE DIFERENTES ESQUEMAS DE PARAMETRIZAÇÃO CUMULUS COM O MODELO REGCM4
}

\author{
Jéssica S. Gonçalves, Simone E. T. Ferraz \\ Universidade Federal de Santa Maria - Programa de pós-graduação em Meteorologia \\ jessicastogon@gmail.com
}

\section{RESUMO}

Neste artigo é feita uma avaliação de diferentes esquemas de parametrização cumulus a fim de descobrir qual parametrização representa melhor a região de Santa Maria-RS no período FMA/2013. Através do diagrama de Taylor pode ser visto que os esquemas Emanuel, Grell FC Dry e AS Wet, Emanuel oceano e Grell FC terra Dry e Wet foram os que melhor representaram a região.

\begin{abstract}
In this paper some evaluations of cumulus parameterization schemes are done to see which best fits the region of Santa Maria-RS the period FMA/2013. Through the analysis it can be seen that the schemes of Emanuel, Grell FC Dry and AS Wet, Emanuel ocean and Grell land FC Dry and Wet were the ones that best represent the region.
\end{abstract}

\section{INTRODUÇÃO}

Uma boa previsão climática de precipitação depende da capacidade do modelo em resolver processos de sub-grade, por isso é empregado o uso de Modelos Climáticos Regionais (MCR) ao invés de Modelos Globais. Apesar de MCR serem recomendados para prever precipitação, é necessário avaliar qual parametrização cúmulos (PC) representa melhor a região analisada. Para isso, utilizamos técnicas estatísticas para comparar os dados simulados e observados a fim de validar o desempenho do MCR na representação do clima local. Neste trabalho, foi utilizado o modelo RegCM4 (Giorgi et al., 2012) para avaliar qual PC representa melhor o clima da região de Santa Maria - RS no período de FMA/2013.

\section{METODOLOGIA}

Os esquemas de PC avaliados foram (tabela 1): Grell (Grell, 1993) que considera a nuvem com duas circulações em equilíbrio, uma ascendente e outra descendente. $\mathrm{O}$ entranhamento ocorre somente na parte superior e inferior da nuvem, sendo o fluxo de massa constante no interior. O esquema é acionado quando uma parcela de ar é levantada e atinge o Nível de Condensação por Levantamento. Duas hipóteses de fechamento podem ser utilizadas: AS74 (Arakawa \& Schubert) e FC80 (Fritsch \& Chappell); e MIT-Emanuel (Emanuel, 1991; Emanuel e Zivkovic-Rothman, 1999) assume que a mistura de nuvens é altamente episódica e não homogênea e considera os fluxos convectivos baseado em um modelo idealizado de correntes ascendentes e descendentes. A convecção é acionada quando o nível de equilíbrio é maior do que o nível de base da nuvem. 
Tabela 1 - Esquemas de parametrização cúmulos

\begin{tabular}{lllll}
\hline$N^{\circ}$ & Parametrização & Grell param & $\begin{array}{l}\text { Peff } \\
\text { Mín. }\end{array}$ & Máx. \\
\hline 01 & Emanuel & -- & - & - \\
07 & Grell FC & Dry & 0,25 & 1,00 \\
10 & Emanuel terra e Grell FC oceano & Dry & 0,25 & 1,00 \\
13 & Emanuel oceano e Grell FC terra & Dry & 0,25 & 1,00 \\
19 & Emanuel terra e Grell FC oceano & Wet & 0,25 & 0,50 \\
22 & Emanuel oceano e Grell FC terra & Wet & 0,25 & 0,50 \\
31 & Emanuel oceano e Grell AS terra & Dry & 0,25 & 1,00 \\
34 & Grell AS & Wet & 0,25 & 0,50 \\
37 & Emanuel terra e Grell AS oceano & Wet & 0,25 & 0,50
\end{tabular}

Grell param é um parâmetro do esquema Grell que permite controlar a fração da precipitação evaporada na corrente descendente - Peff, escolhendo dry temos Peff entre 0,25 e 1,0 que aumenta a fração de evaporação e escolhendo wet temos Peff entre 0,25 e 0,50 que diminui a fração de evaporação.

$\mathrm{Na}$ avaliação dos esquemas de PC, foi utilizado o diagrama de Taylor (2001) que consiste em um diagrama que compara um conjunto de dados $f_{n}$ (previsão) a uma referência $r_{n}$ (dados de precipitação das estações automáticas do INMET de Santa Maria) através das estatísticas:

$\checkmark$ Raiz do erro quadrático centrado na média (E) (Eq.5).

$\checkmark$ Desvio Padrão $(\sigma)$ (Eq.6 e 7).

$\checkmark$ Coeficiente de Correlação de Pearson (R) (Eq.8).

As estatísticas são relacionadas através da lei dos cossenos, podendo ser representadas em um mesmo diagrama através da Eq.9.

$$
\begin{aligned}
& \sigma_{\mathrm{f}}^{2}=\frac{1}{\mathrm{~N}} \sum_{1}^{\mathrm{N}}\left(\mathrm{f}_{\mathrm{n}}-\overline{\mathrm{f}}\right)^{2} \\
& \sigma_{\mathrm{r}}^{2}=\frac{1}{\mathrm{~N}} \sum_{1}^{\mathrm{N}}\left(\mathrm{r}_{\mathrm{n}}-\overline{\mathrm{r}}\right)^{2}
\end{aligned}
$$

$$
\mathrm{R}=\frac{\frac{1}{\mathrm{~N}} \sum_{1}^{\mathrm{N}}\left(\mathrm{f}_{\mathrm{n}}-\overline{\mathrm{f}}\right)\left(\mathrm{r}_{\mathrm{n}}-\overline{\mathrm{r}}\right)}{\sigma_{\mathrm{f}} \sigma_{\mathrm{r}}}
$$

$$
c^{2}=\sigma_{\mathrm{f}}^{2}+\sigma_{\mathrm{r}}^{2}-2 \sigma_{\mathrm{f}} \sigma_{\mathrm{r}} R
$$

No diagrama cada conjunto de dados é representado por um ponto que é definido conforme os valores de correlação, desvio padrão e Raiz do erro quadrático centrado na média. Quanto mais próximo os pontos estiverem maior o grau de similaridade entre eles.

\section{RESULTADOS}

Através do diagrama de Taylor podemos ver que os experimentos 01, 07, 13, 22 e 34 foram os que representaram melhor a região de Santa Maria para o período analisado. 


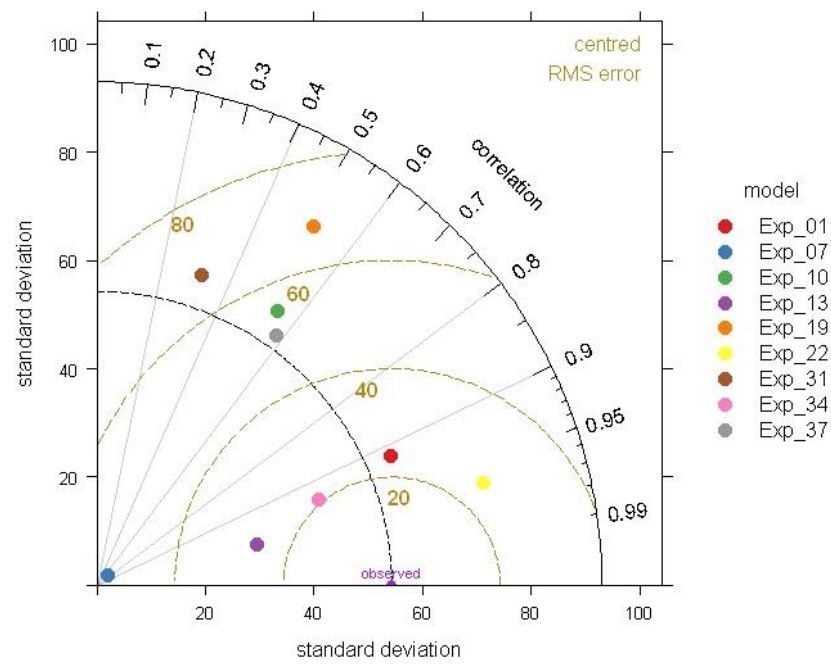

Figura 1 - Diagrama de Taylor. Comparação estatística entre nove previsões sazonais com parametrizações cúmulos diferentes e os dados observados do INMET no período de fevereiro, março e abril.

\section{CONCLUSÕES}

Através do diagrama de Taylor, pode ser visto que os esquemas Emanuel, Grell FC Dry e AS Wet, Emanuel oceano e Grell FC terra Dry e Wet foram os que melhor representaram a região de Santa Maria-RS no período de fevereiro, março e abril de 2013.

\section{AGRADECIMENTOS}

Agradeço ao CNPq pelo apoio, a CAPES pela bolsa de pós-graduação, ao CRS/INPE pela infra-estrutura e a UFSM pela oportunidade.

\section{BIBLIOGRAFIA}

EMANUEL, K. A. A Scheme for Representing Cumulus Convection in Large-Scale Models. Journal of Atmospheric Sciences, Washington DC, v. 48, n. 21, p. 2313-2329, 1991. EMANUEL, K. A.; ZIVKOVIC-ROTHMAN, M. Development and Evaluation of a Convection Scheme for Use in Climate Models. Journal of Atmospheric Sciences, Washington DC, v. 56, n. 11, p. 1766-1782, 1999.

GIORGI, F. et al. RegCM4: model description and preliminary tests over multiple CORDEX domains. Climate Research: CR Special 29, Oldendorf, v. 52, p. 7-29, 2012.

GRELL, G. A. Prognostic Evaluation of Assumptions Used by Cumulus Parameterizations. Monthly Weather Review, Washington DC, v. 121, n. 3, p. 764-787, 1993.

TAYLOR, K. E. Summarizing multiple aspects of model performance in a single diagram. Journal of Geophysical Research, Washington DC, v. 106, n. D7, p. 7183-7192, 2001. 